\title{
Visibilidade da enfermagem sob a ótica de universitários
}

Recebido em 21/11/2012

Aprovado em 16/05/2014
Liziani Iturriet Avila' Rosemary Silva da Silveira ${ }^{2}$ Juliana Teixeira da Silveira ${ }^{3}$

Valéria Lerch Lunardi ${ }^{4}$

Lisiane Van Ommeren ${ }^{5}$

Geani Farias Machado Fernandes ${ }^{6}$

Resumo: Objetivou-se conhecer as percepções de universitários acerca da visibilidade da enfermagem. Utilizou-se uma abordagem qualitativa do tipo exploratória, através da técnica de entrevista com dezenove universitários dos Cursos de Enfermagem, Medicina, Educação Física e Psicologia de uma Universidade Federal do sul do país. Os dados foram submetidos à Análise Textual Discursiva. Buscar as percepções junto aos universitários de diferentes cursos proporcionou evidenciar visões distintas sobre a enfermagem e sua visibilidade, oportunizando uma reflexão acerca do fazer desses profissionais. Descritores: Enfermagem, Universitários, História da enfermagem

\section{Nursing visibility under the view of undergraduate students}

Abstract: We aimed to find out the perceptions of undergraduate students regarding the visibility of the nursing course. A qualitative and exploratory approach was applied, through the technique of interviews with nineteen undergraduate students of the courses of Nursing, Medicine, Physical Education and Psychology in a Federal University in southern Brazil. Data were submitted to analysis under the discursive textual analysis. Seeking perceptions among undergraduate students of different courses led us to distinguished views concerning the nursing course and its visibility, providing us with meaningful considerations towards the practices of these professionals.

Descriptors: Nursing, Undergradute Students, History of nursing

\section{Visibilidad de la enfermería sob la perspectiva de los académicos}

Resumen: Se ha objetivado conocer las percepciones de los estudiantes acerca de la visibilidad de la enfermería. Se ha utilizado un enfoque cualitativo exploratorio, a través de la técnica de encuesta con diecinueve académicos de los cursos de Enfermería, Medicina, Educación Física y Psicología de la Universidad Federal del sur de Brasil. Los datos fueron sometidos a análisis textual discursivo. Buscar las percepciones entre los estudiantes de distintos cursos ha proporcionado destacar distintos puntos de vista acerca de la enfermería y su visibilidad, dando la oportunidad de una reflexión sobre del hacer de estos profesionales.

Descriptores: Enfermería, Historia de la Enfermería

\section{INTRODUÇÃO}

A enfermagem possui um significativo impacto na qualidade da assistência em saúde; representando aproximadamente $60 \%$ do conjunto das profissões de saúde, superando o marco de mais de um milhão e trezentos mil trabalhadores; atuando na rede hospitalar durante as 24 horas de todos os dias do ano ${ }^{(1,2)}$.

Apesar de sua expressiva qualificação e intensificação na produção de conhecimentos para a "formação de um corpo de conhecimentos científicos próprios" e a "sua definição como ciência", ainda é visível em diversos segmentos da sociedade e da mídia certa invisibilidade e ausência de clareza do fazer da enfermagem ${ }^{(3: 583)}$.

É provável que as distorções acerca da imagem da enfermagem sejam decorrentes da ausência de conhecimento existente no processo formativo de universitários que farão parte de equipes multiprofissionais de saúde, repercutindo em comportamentos ainda vigentes nas instituições, sustentando a invisibilidade da enfermagem.

É possível inferir que a maioria dos estudiosos dessa temática realizou pesquisas priorizando a visão dos profissionais da comunicação $^{(4)}$, da equipe de enfermagem ${ }^{(5)}$, do paciente ${ }^{(6)}$, de acadêmicos do curso de enfermagem ${ }^{(7)}$, dentre outros. Assim, acredita-se que as percepções de universitários de diferentes cursos acerca da visibilidade da enfermagem podem evidenciar outras visões para a melhor compreensão do problema estudado. Objetivou-se conhecer as percepções de universitários acerca da visibilidade da Enfermagem.

'Enfermeira. Aluna do Doutorado em Enfermagem da Universidade Federal do Rio Grande. Bolsista CAPES

${ }^{2}$ Enfermeira. Doutora em Enfermagem/ UFSC. Docente da Escola de Enfermagem e do Programa de Pós Graduação em Enfermagem da Universidade Federal do Rio Grande/FURG.

${ }^{3}$ Enfermeira. Mestre em Enfermagem do Programa de Pós-Graduação em Enfermagem da Universidade Federal do Rio Grande.

${ }^{4}$ Enfermeira. Doutora em Enfermagem/ UFSC. Docente do Programa de Pós Graduação em Enfermagem da Universidade Federal do Rio Grande/FURG.

${ }^{5}$ Enfermeira. Aluna do Mestrado em Enfermagem pela Universidade Federal do Rio Grande.

${ }^{6}$ Enfermeira. Doutora em Enfermagem/ UFSC. Docente da Escola de Enfermagem da Universidade Federal do Rio Grande/FURG. 


\section{METODOLOGIA}

Trata-se de uma pesquisa de abordagem qualitativa, do tipo exploratório. Utilizou-se como técnica de coleta de dados a entrevista composta por questões semi-estruturadas ${ }^{(8)}$ Participaram dezenove universitários dos Cursos de Enfermagem, Medicina, Educação Física e Psicologia, de uma Universidade Federal do extremo sul do país, regularmente matriculados no último ano. Para preservar seu anonimato, foram identificados pelas letras: $E$ (enfermagem), $M$ (medicina), $P$ (psicologia) e EF (educação física), seguidas de numeração arábica, de acordo com a ordem das entrevistas. Obteve-se a aprovação do Comitê de Ética em Pesquisa na Área da Saúde, mediante o Parecer 117/2010, contemplado a Resolução 196/96(9).

O processo de análise dos dados foi realizado a partir da Análise Textual Discursiva constituída por 4 passos: "desmontagem do texto" - as transcrições foram separadas em unidades de significado formando o corpus; "estabelecimento de relações" - processo de categorização das unidades por semelhança; "captar o novo emergente" e "um processo auto organizado" - descrição e interpretação dos significados construídos a partir do corpus, expressando novos entendimentos sobre o fenômeno investigado ${ }^{(10)}$.

\section{RESULTADOS}

1. O conhecimento dos Universitários acerca do trabalho da enfermagem

O desconhecimento do trabalho da enfermagem é evidenciado no processo de formação acadêmica dos diferentes cursos estudados:

Como acadêmico tenho uma visão superficial da enfermagem dentro da equipe! Depois de formado vou ver. (M1)

a valorização da enfermagem tem que partir dos próprios profissionais [...] poderia estar melhor se começasse desde a formação dos profissionais que iriam trabalhar com o enfermeiro. (P4)

Do mesmo modo, os universitários desconhecem a diferenciação das categorias profissionais da enfermagem e suas atribuições:

a divisão de trabalho é feita entre técnicos e enfermeiros [...] Sobre os auxiliares realmente não sei. (E1)

Entre técnico e auxiliar deve haver uma diferença de formação, mas não sei identificar. $O$ enfermeiro tem graduação e, acredito que devido a isso, as demandas, habilidades e autonomia no trabalho de cada um sejam diferentes. (P5)

na maioria das unidades todos parecem fazer a mesma coisa. (EF1)

Apesar do aparente desconhecimento acerca do trabalho da enfermagem, os universitários manifestaram interesse em conhecer as diversidades de cada profissão durante seu processo formativo:

Durante a graduação deveriam informar sobre as atividades dos futuros profissionais; por exemplo, como psicólogo tenho possibilidade de trabalhar [...] com médicos, enfermeiros, fisioterapeutas [...] e o que cada um faz? (P5).

\section{A percepção dos Universitários acerca da visibilidade midiática e social da enfermagem}

Na concepção dos universitários, a imagem social do ser enfermeiro reproduzida na mídia, na maioria das vezes, está associada à figura feminina:

na cena, se aparece uma mulher é a enfermeira e, se aparece um homem é o médico. (P5)

Outro aspecto ressaltado pelos universitários refere-se à imagem de submissão do enfermeiro aos outros profissionais da área da saúde, frequentemente veiculada na mídia:

as novelas mostram que o enfermeiro não tem autonomia, fica do lado do médico só obedecendo e não é assim que é o enfermeiro. (E3)

Na novela, chega um médico na cidade e contrata alguém para trabalhar de assistente dele, o "enfermeiro" (sinalizou aspas) qualquer pessoa que não sabe medicina, mas que faz o que o médico não está disposto a fazer. (P4)

Um aparente desconhecimento da mídia com a profissão de enfermagem que se expressa como desrespeito reforça um estereótipo negativo no imaginário da população:

a mídia vê o profissional da enfermagem como todos da mesma categoria [...] veicula de forma errada [...] a população não conhece quem é o enfermeiro, quem é o técnico, quem é $o$ auxiliar. (M3)

Olha, a mídia reproduz pouco a imagem do enfermeiro, e quando aparece, o enfermeiro rouba bebês; dopa os pacientes; é vilã, dançarina, sensual; não vejo propaganda positiva do enfermeiro. (EF1)

A erotização da imagem da enfermeira, reproduzida na mídia, contribui para deturpar e representar pejorativamente a profissão:

aparece muito a erotização da mulher enfermeira (P4)

é imoral a imagem reproduzida na mídia [...] a enfermeira é sensual, apresenta peitos salientes, usa mini saia [...] é vergonhoso, ninguém toma providências para modificar essa desmoralização com a profissão. (E4)

\section{Propostas para a valorização da enfermagem}

Ao refletirem sobre as dificuldades enfrentadas pela enfermagem, os universitários expressaram que a sua valorização é um desafio para os próprios trabalhadores da enfermagem e para os órgãos representativos da profissão, sendo necessário realizar o marketing pessoal para promover a profissão, valorizando a imagem profissional através da postura que cada um assume no exercício da enfermagem.

Mostrar que tens conhecimento [...] que a enfermagem é diferente das demais e cada profissão tem o seu valor; tentar ocupar o teu espaço. (E4)

Para que os outros cursos passem a valorizar a enfermagem tem que ter um trabalho da própria equipe, do curso de 
formação acadêmica para que eles tenham uma dimensão do seu valor. (M5)

Uma das formas de valorizar e aumentar a visibilidade da enfermagem está relacionada à postura que o enfermeiro assume como coordenador e líder da equipe:

O enfermeiro é o aparelho locomotor [...] ele dá sustentação e capacidade de movimento [...] está mais imerso no funcionamento do hospital, no contato direto com o paciente, no conhecimento dos vários âmbitos da instituição porque gerencia a unidade que trabalha, isso dá visibilidade ao seu trabalho. (P2)

Além do gerenciamento e a capacidade de liderança, outro aspecto destacado para contribuir com a valorização da enfermagem refere-se à realização do Processo de Enfermagem:

Registrar o que se faz, prescrever cuidados, evoluir pacientes é uma forma de valorizar mais, se não documentarmos parece que a enfermagem não faz nada. (E5)

Os entrevistados evidenciaram que a valorização da enfermagem pela mídia pode constituir-se numa estratégia para promover a visibilidade e o reconhecimento da profissão:

Com o poder de influência social que a mídia tem, se mostrassem o valor da enfermagem, o conhecimento e participação na equipe, a população teria outra visão da enfermagem. (M6

A mídia deveria mostrar mais a enfermeira como ela realmente é, mas para isso, o órgão de classe de vocês deveria informar a mídia, instrumentalizar a mídia, entende? (EF1)

\section{DISCUSSÃO}

Para promover a qualidade do cuidado e a eficácia dos serviços, a equipe multidisciplinar de saúde deve conhecer as atribuições de cada profissão, respeitando o espaço de cada um e trocando experiências entre $\mathrm{si}^{(11)}$. No entanto, esse conhecimento não está presente no processo de formação dos cursos estudados, o que demonstra a necessidade de inserir essas questões durante a formação acadêmica dos trabalhadores da saúde e, prioritariamente, da enfermagem, pois mesmo os acadêmicos de Enfermagem apresentaram desconhecimento e dificuldades em identificar as atribuições de cada categoria.

O trabalho da enfermagem é realizado por diferentes categorias profissionais, as quais apresentam um grau de escolaridade diferente, que pressupõe um conjunto distinto de atribuições profissionais, que correspondem a "um processo de formação próprio"(12:425). A Lei no 7.498, de 25 de junho de 1986 dispõe sobre a regulamentação do exercício profissional da enfermagem estabelecendo as atribuições legais para enfermeiros, técnicos e auxiliares de enfermagem. Apesar dessa legislação, parece haver uma prática não suficientemente diferenciada entre os trabalhadores de enfermagem, o que de certo modo, contribui para o desconhecimento dos sujeitos desse estudo acerca dessas diferenças ${ }^{(13)}$.

Os universitários percebem a relevância da interação da equipe multiprofissional, destacando a necessidade de "conhecer o que cada um faz" durante o processo de formação para promover a interação dos futuros trabalhadores da área da saúde, a comunicação, o respeito, a compreensão das diferenças das atribuições de cada categoria, o que requer: "a necessidade das relações sustentarem-se na cooperação e na troca entre as disciplinas, na interação entre os profissionais, na articulação dos saberes e fazeres, na horizontalização das relações e na participação na tomada de decisões com base na construção de espaços para a elaboração e expressão de subjetividades"(14:865).

No que tange à percepção acerca da visibilidade midiática e social da enfermagem foi possível evidenciar que a figura feminina é veiculada associada ao trabalho da enfermagem, enquanto que o homem é relacionado ao fazer do médico. A despeito do espaço ocupado pelas mulheres enfermeiras, prevalece ainda uma imagem antiga de subordinação da mulher ao homem e da enfermagem à medicina. No entanto, apesar da enfermagem ser constituída majoritariamente por mulheres; o papel social feminino tem mudado no decorrer das décadas e a figura que, antes era frágil, atualmente assume uma postura de destaque na sociedade ${ }^{(15)}$.

Além da imagem de submissão do enfermeiro e sua associação à figura feminina, é frequente a veiculação de um estereótipo negativo e pejorativo. De um modo geral, a mídia, em especial as telenovelas, veicula informações que exploram o corpo da mulher enfermeira como símbolo sexual, estigmatizando sua imagem. Na internet, é comum encontrar figuras erotizadas associadas à palavra enfermeira ou enfermagem. Essa erotização da profissão é uma das deturpações que aparecem no imaginário popular, provavelmente devido às relações com o passado histórico da enfermagem, que, inicialmente, era "exercida por mulheres de moral duvidosa (prostitutas, alcoolistas, analfabetas)", que desenvolviam seu fazer de modo submisso(16:69).

No que tange às propostas para a valorização da profissão, os universitários referiram que os trabalhadores da enfermagem devem desenvolver o marketing pessoal, não somente relacionado à aparência pessoal, mas a postura exercida na profissão. O marketing do enfermeiro pode ser construído a partir do exercício de sua autoridade, da demonstração de domínio do conhecimento, do exercício competente da prática profissional, compartilhando responsabilidades, tomando decisões, exigindo condições necessárias de trabalho, organizando o trabalho da equipe, o que inclui o gerenciamento do fazer da enfermagem ${ }^{(17,18)}$.

Nessa perspectiva, os universitários consideraram que a valorização da enfermagem pode ser conquistada a partir do modo como os enfermeiros gerenciam seu fazer; exercem sua autonomia; ampliam seu espaço de decisão, e, ainda, como assumem sua posição em relação aos demais profissionais da saúde, pois "recebem conotação de poder e, de alguma forma, afastam o desgastado rótulo de submissão"(19:39).

Além do gerenciamento, outro aspecto destacado que pode contribuir para a credibilidade e visibilidade da enfermagem refere-se à concretização do Processo de Enfermagem, um instrumento metodológico que orienta o cuidado de Enfermagem e documenta a prática profissional. Ao realizar o Processo de Enfermagem, o enfermeiro além de cumprir uma exigência legal, contempla, também, o direito do usuário de ter seus diagnósticos de enfermagem evidenciados, com a intervenção de enfermagem avaliada e organizada por quem possui conhecimento para tal. Assim, o enfermeiro pode exercer sua autonomia profissional e obter mais satisfação para si, para a equipe e o usuário, resultando na visibilidade 
das ações realizadas pela enfermagem ${ }^{(20)}$.

A mídia, na visão dos universitários, constitui-se num possível instrumento para ampliar o conhecimento da população sobre a enfermagem, podendo modificar o panorama pejorativo existente acerca dessa profissão, valorizando o seu fazer, promovendo sua visibilidade e reconhecimento.

\section{CONCLUSÕES}

Evidenciou-se que há um desconhecimento acerca do trabalho da enfermagem por parte de universitários dos diferentes cursos. Os universitários consideram que o fornecimento de informações acerca das atribuições profissionais dos diferentes membros da equipe multidisciplinar, desde a sua formação acadêmica, pode fortalecer suas relações de trabalho, assim como o reconhecimento e a valorização das profissões.

A imagem que é veiculada na mídia acerca da enfermagem é distorcida, pois é visualizada como uma profissão subordinada a outros profissionais. A mídia reproduz uma imagem estereotipada, transmitindo a ideia de não-cientificismo da profissão; explorando o corpo da mulher enfermeira como símbolo sexual; difundindo uma imagem de submissão, aparentemente negando sua existência e importância, valorizando apenas outros profissionais da saúde, e a população parece absorver essa ideia falsa.

Provocar mudanças na imagem da profissão, provavelmente, exija a veiculação, de forma verossímil, da Enfermagem, instrumentalizando os profissionais da comunicação acerca do conhecimento sobre essa profissão, punindo a comunicação de inverdades divulgadas. Se a mídia apresentasse ideias positivas acerca da Enfermagem, provavelmente, haveria uma mudança na opinião pública, com ampliação da valorização e da credibilidade profissional.

\section{Referências}

1. Brasil. Ministério do Trabalho e Emprego (BR). Relação Anual de Informações Sociais/RAIS, Brasília: Ministério do Trabaho; 2008.

2. Pires D. A enfermagem enquanto disciplina, profissão e trabalho. Rev Bras Enferm. Brasília 2009 set-out; 62(5): 739-44.

3. Padilha MICS, Borenstein MS. O método de pesquisa histórica na enfermagem. Texto Contexto Enferm, Florianópolis, 2005 Out-Dez; 14(4):575-84.

4. Kemmer LF, Silva MJP. A visibilidade do enfermeiro segundo a percepção de profissionais de comunicação. Rev. Latino-Am. Enfermagem. 2007, 2(15):191-198.

5. Castanha ML, Zagonel IPS. A prática de cuidar do ser enfermeiro sob o olhar da equipe de saúde. Rev. bras. enferm. 2005; 5(58):556-562.

6. Santos CB, Luchesi LB. A imagem da enfermagem frente aos estereótipos: uma revisão bibliográfica. Simp. Bras. Comun. Enferm. Maio, 2002, São Paulo.

7. Erdmann AL, Fernandes JV, Melo C, Carvalho BR, Meneses Q, Freitas R, Emarinony E, Backesi MTS. A visibilidade da profissão de enfermagem: reconhecendo conquistas e lacunas. Rev. bras. enferm. 2009, 4(62):637-643.

8. Polit DF, Beck CT. Fundamentos de pesquisa em enfermagem: avaliação de evidências para a prática da enfermagem. Porto Alegre: Artmed, 2011.

9. Ministério da Saúde. Conselho Nacional de Saúde. Resolução № 196/96. Dispõe sobre pesquisa envolvendo seres humanos. Brasília, 1996.

10. Moraes R, Galiazzi MC. Análise Textual Discursiva. Ijuí: Ed Unijuí; 2011.

11. Pinho M C. G. Trabalho em equipe de saúde: limites e possibilidades de atuação eficaz. Programa de pós-graduação em psicologia social - UERJ. Rio de Janeiro: Brasil: 2006.

12. Peduzzi M, Anselmi ML. O auxiliar e o técnico de enfermagem: categorias profissionais diferentes e trabalhos equivalentes. Rev. bras. enferm. 2004: 4(57):425-429.

13. Brasil. Lei n 7.498 , de 25 de junho de 1986 dispõe sobre a Regulamentação do Exercício Profissional da Enfermagem.

14. Matos E, Pires, DEP, Campos GWS. Relação de trabalhadores em equipe interdisciplinares: contribuições para a construção de novas formas de organização do trabalho em saúde. Rev. Brasil Enferm. Brasília 2009. nov-dez; 62(6):863-869.

15. Assunção LA, Costa CSC, Lourenço HF. Mitos de Enfermagem: Mulher, Trabalho e Gênero. I Congresso Internacional Sobre a Mulher, Gênero e Relações de Trabalho, 2005. Goiânia - GO

16. Colpo JC, Camargo VC, Mattos SA. A Imagem Corporal da Enfermeira como Objeto Sexual na Mídia: um assédio a profissão. Cogitare Enferm. 2006. jan/abr; 11(1):67-72.

17. Gentil RC. O enfermeiro não faz marketing pessoal: a história explica por quê? Revista Brasil Enferm. 916-918. Brasília - DF. 2009.

18. Silveira RS. A construção moral do trabalhador de saúde como sujeito autônomo e ético. (Tese Doutorado em Enfermagem) Florianópolis: Universidade Federal de Santa Catarina; 2006.

19. Brito AMR. Representações sociais de discentes de Enfermagem sobre ser enfermeiro. (Dissertação de Mestrado em Enfermagem) Minas Gerais: Universidade Federal de Minas Gerais; 2008.

20. Brasil. Resolução Cofen-358/2009. Dispõe sobre a Sistematização da Assistência de Enfermagem e a implementação do Processo de Enfermagem em ambientes, públicos ou privados. 